\section{Assessment of feline blood pressure in primary care practice - The Mercury Challenge, a European survey}

\section{Andrew Sparkes ${ }^{1}$, Catherine Garelli-Paar², Emilie Guillot ${ }^{2}$}

1 Simply Feline Veterinary Consultancy, Shaftesbury, United Kingdom

2 Ceva Sant Animale, Libourne, France

\section{OBJECTIVES}

Although systemic hypertension is an important and common condition in cats, there is a paucity of information on blood pressure (BP) values typically measured in cats attending primary care practices. This convenience survey was designed to collect BP data from a large number of cats across numerous European countries.

\section{METHODS}

From June 2018, Ceva Santé Animale invited primary care clinics to record systolic BP (SBP) data from cats aged $\geq 7$ years who had BP measured as part of recognised veterinary practice. Owners granted permission to record anonymised information from the cats on a central database (mercurychallenge.ceva.com), including basic demographic data, information on concomitant disease and/or medications, SBP values, and any antihypertensive therapy given.

\section{RESULTS}

By May 2019, data from $>6000$ cats across 10 European countries revealed that in most $(91 \%)$ cases it took $\leq 10$ minutes to measure SBP, and during the procedure $49 \%$ cats were described as 'calm', and $42 \%$ as 'cooperative but anxious'. In 94\%, SBP was measured via Doppler or oscillometric equipment and recorded mean SBP values were $<140 \mathrm{mmHg}$ in $37.3 \%, 140-159$ in $28.5 \%$, $160-179$ in $17.0 \%$ and $\geq 180$ in $17.2 \%$. Chronic kidney disease (CKD) was more prevalent in cats with SBP $\geq 160$ $(30 \%)$ than in cats with SBP $<160(19 \%) \mathrm{P}<0.0001$

\section{STATEMENT (CONCLUSIONS)}

The results of this survey suggested a high prevalence of cats with potential hypertension and that SBP could be readily measured in most cats in primary care practice. Cats reported to have CKD had significantly higher mean SBP values than those without.

\section{The first Core}

Outcome Set for cats: the most important parameters to assess in feline CKD treatment

\section{Hannah Doit ${ }^{1}$, Rachel Dean ${ }^{2}$, Marco Duz ${ }^{1}$, Richard Emes ${ }^{1}$, Marnie Brennan ${ }^{1}$}

1 University of Nottingham, Nottingham, United Kingdom

2 Vet Partners, York, United Kingdom

\section{OBJECTIVES}

Core Outcome Sets (COS) are recommended for inclusion in future treatment trials so that the results of future research can be compared and combined. The aim of this work was to finalise the COS for cats with chronic kidney disease (CKD).

\section{METHODS}

An international panel of 16 stakeholders, including cat owners, clinical experts and regulatory agencies, participated in a one-day chaired consensus meeting Previous research using a Delphi approach had resulted in a baseline of 27 parameters for inclusion. Twenty borderline parameters which had the greatest disagreement between different stakeholder groups were addressed. Each was clearly defined, the importance of inclusion debated and then voted on. Consensus was defined a priori as $80 \%$ of stakeholders voting it 8 or 9 out of 9 Finally, all parameters were streamlined and amalgamated by discussion and voting, into common core areas.

\section{RESULTS}

The final number of parameters after the consensus meeting was 29. These were grouped into 9 core parameter areas: clinical examination, food, blood biochemistry, complete blood count, urine tests, quality of life, survival, progression of CKD and adverse events.

\section{STATEMENT (CONCLUSIONS)}

The final COS is recommended for inclusion in all future treatment trials for cats with CKD as it represents the parameters of importance to all those making treatment decisions for these patients in both first opinion and referral practice. This is the second veterinary COS to be created, and the first $\operatorname{COS}$ to be created for feline patients. 